\title{
Analysis of Circularly Polarized Hemispheroidal Dielectric Resonator Antenna Phased Arrays Using the Method of Auxiliary Sources
}

\section{Larsen, Niels Vesterdal; Breinbjerg, Olav}

Published in:

I E E E Transactions on Antennas and Propagation

Link to article, DOI:

10.1109/TAP.2007.900257

Publication date:

2007

Document Version

Publisher's PDF, also known as Version of record

Link back to DTU Orbit

Citation (APA):

Larsen, N. V., \& Breinbjerg, O. (2007). Analysis of Circularly Polarized Hemispheroidal Dielectric Resonator Antenna Phased Arrays Using the Method of Auxiliary Sources. I E E E Transactions on Antennas and Propagation, 55(8), 2163-2173. https://doi.org/10.1109/TAP.2007.900257

\section{General rights}

Copyright and moral rights for the publications made accessible in the public portal are retained by the authors and/or other copyright owners and it is a condition of accessing publications that users recognise and abide by the legal requirements associated with these rights.

- Users may download and print one copy of any publication from the public portal for the purpose of private study or research.

- You may not further distribute the material or use it for any profit-making activity or commercial gain

- You may freely distribute the URL identifying the publication in the public portal 


\title{
Analysis of Circularly Polarized Hemispheroidal Dielectric Resonator Antenna Phased Arrays Using the Method of Auxiliary Sources
}

\author{
Niels Vesterdal Larsen and Olav Breinbjerg, Member, IEEE
}

\begin{abstract}
The method of auxiliary sources is employed to model and analyze probe-fed hemispheroidal dielectric resonator antennas and arrays. Circularly polarized antenna elements of different designs are analyzed, and impedance bandwidths of up to $14.7 \%$ are achieved. Selected element designs are subsequently employed in a seven-element phased array. The array performance is analyzed with respect to scan loss and main beam directivity as a function of scan angle and frequency, and the influence of element separation is investigated.
\end{abstract}

Index Terms-Dielectric resonator antennas (DRAs), method of auxiliary sources, phased arrays.

\section{INTRODUCTION}

$\mathbf{T}$ HE dielectric resonator antenna (DRA) has attracted much attention in recent years due to its many favorable features such as low loss, compact size, structural simplicity, and simple feeding schemes. Various different shapes have been investigated, the most common being hemispherical, cylindrical, and rectangular shapes, e.g., [1]-[4].

In order to obtain a large bandwidth, a wide range of complex shapes have been used, common to which is the inclusion of an air gap inside the DRA [5]-[7]. Also multiple-band operation has been achieved by using inhomogeneous dielectrics in the form of stacked DRAs or by enclosing one DRA in another [8], [9].

Circularly polarized DRAs have been designed using either single-feed or multiple-feed excitations. With a single feed, orthogonal modes can be excited in the DRA by applying parasitic patches [10] or by exciting the DRA asymmetrically [11]. These methods, however, are generally very narrow-band with respect to the axial ratio, and therefore practical designs often make use of multiple feeds excited in phase quadrature, e.g., [12].

The compact size is an important factor when considering the DRA as a candidate for phased arrays. This allows smaller element separation compared with many other antenna types, and this is important for reducing scan loss [13]. Planar linearly polarized DRA phased arrays have previously been examined in [14] and [15]; and in [16] a circularly polarized phased array was

Manuscript received August 22, 2006; revised February 19, 2007.

N. V. Larsen is with the Technical University of Denmark, DK-2800 Kgs. Lyngby, Denmark and also with Thrane \& Thrane, DK-2800 Kgs. Lyngby, Denmark (e-mail:nvl@oersted.dtu.dk).

O. Breinbjerg is with the Technical University of Denmark, DK-2800 Kgs. Lyngby, Denmark.

Digital Object Identifier 10.1109/TAP.2007.900257 developed. In [14], the influence of the mutual coupling between the elements on the radiation pattern was investigated. However, a detailed investigation of the array scan loss and its variation with element distance and frequency has not been reported for these designs.

Much theoretical work has been done in analyzing hemispherical DRA elements where spherical wave expansion (SWE) techniques can be used to derive analytical solutions or be combined with numerical techniques such as the method of moments (MoM) to solve for currents on probes or fields in apertures that excite the DRA, e.g., [1] and [2]. For more general shapes of DRAs or for DRA arrays, accurate modelling usually relies on numerical techniques, e.g., the finite-element method (FEM) or MoM. For arrays in particular, the computational cost of numerical analysis may be prohibitive with these methods.

The method of auxiliary sources (MAS) is well known for its low computational cost and may thus be an alternative to MoM and FEM. Indeed, MAS has previously been employed in the analysis of dielectric antennas [17], where an infinite periodic waveguide array with protruding dielectric elements was investigated. In particular, the simple and computationally cheap standard MAS [18] can be employed when the field varies smoothly along the boundaries, and in this case simple Hertzian dipoles can be used as auxiliary sources. For configurations where the fields vary rapidly near the boundary, e.g., near edges or closely positioned illuminating sources, the MAS model can be augmented with localized MoM patches [19], [20]. MAS has also been proven effective for thin-wire antennas where, by using sinusoidal dipoles as auxiliary sources, the impedance properties of dipole antennas have been evaluated accurately [21].

The purpose of this paper is twofold. First, it is demonstrated that the simple standard MAS model can be employed for detailed and accurate analysis of small finite arrays of smooth hemispheroidal probe-fed DRAs positioned on an infinite, perfectly electrically conducting (PEC), ground plane. Secondly, the model is used to investigate and design a seven-element hexagonal phased array consisting of such DRA elements. The emphasis is put on circularly polarized elements fed in phase quadrature with four probes. A number of different element designs are first investigated, and subsequently the seven-element phased array is analyzed. Examples of array performance in terms of directivity and scan loss are presented, and the dependence on element separation and scan angle is discussed. In order to validate the MAS model, the results are compared with 

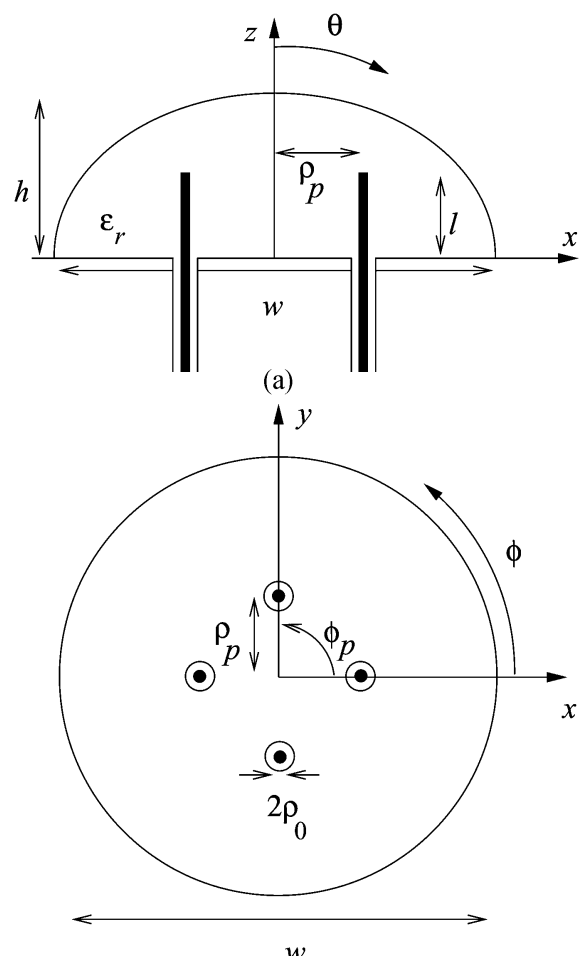

(b)

Fig. 1. Cross-sectional views of the probe-fed hemispheroidal DRA with $P=$ 4 probes. (a) $(x, z)$-plane. (b) $(x, y)$-plane.

those obtained using the software tool CST Microwave Studio (CST-MS) and reference measurement from [22]. In the case of a single hemispherical DRA, comparison is also made with SWE solutions. These validations are done for one- and two-element configurations; however, it was not practically possible to model an entire seven-element array with CST-MS with the available computer resources. This further illustrates the justification of developing this MAS model.

The text is organized as follows. The MAS model is presented in Section II and the element and array investigations are given in Section III and Section IV, respectively. The conclusions are drawn in Section V, and additional mathematical details of the MAS model and the SWE solution are given in Appendixes I and II, respectively.

\section{MAS MODELS OF THE DRA ElEMENTS AND ARRAYS}

\section{A. The DRA Element}

The hemispheroidal DRA element is depicted in Fig. 1, where also the coordinate system is defined. It is positioned on an infinite ground plane and is uniquely described by its height $h$ and width $w$. It is fed by a number $P$ of probes positioned inside the DRA. The position of the $p$ th probe in the $(x, y)$-plane is given by the circular cylindrical coordinates $\left(\rho_{p}, \phi_{p}\right)$, and the probe radius is denoted $\rho_{0}$.

The MAS model of a single DRA with one probe is shown in Fig. 2(a). The upper half-space is divided into two regions: Region 0 outside the DRA and Region 1 inside with the boundary denoted $B$. The outward unit normal vector to $B$ is denoted $\hat{\mathbf{n}}$. The permittivities and permeabilities of the two regions are

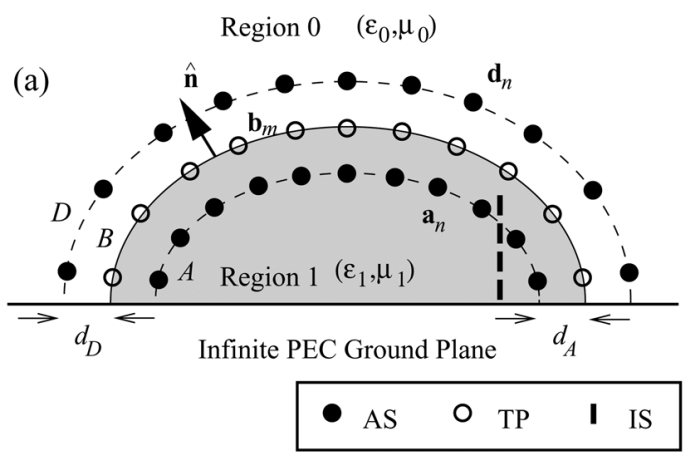

(b) Region $0 \quad\left(\varepsilon_{0}, \mu_{0}\right)$

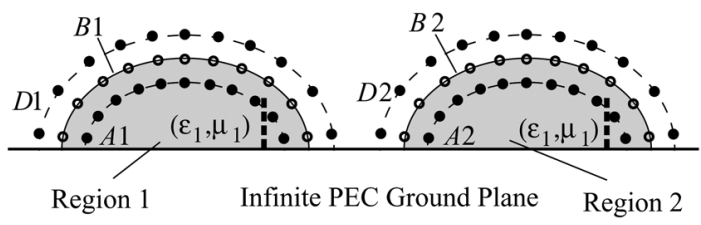

Fig. 2. Cross-section of the MAS model showing ASs, TPs, and ISs for a single-probe configuration. (a) A single DRA element. (b) Two DRA elements.

$\left(\epsilon_{0}, \mu_{0}\right)$ and $\left(\epsilon_{1}=\epsilon_{r} \epsilon_{0}, \mu_{1}=\mu_{r} \mu_{0}\right)$, respectively, where $\epsilon_{r}$ and $\mu_{r}$ are the relative permittivity and permeability of the DRA material. Thus the wave numbers and intrinsic impedances of the two regions are, respectively, $k_{0}=\omega \sqrt{\epsilon_{0} \mu_{0}}, Z_{0}=\sqrt{\mu_{0} / \epsilon_{0}}$ and $k_{1}=k_{0} \sqrt{\epsilon_{r} \mu_{r}}, Z_{1}=Z_{0} \sqrt{\mu_{r} / \epsilon_{r}}$ with $\omega$ being the angular frequency. The total field in the two regions is denoted by $\left(\mathbf{E}_{0}, \mathbf{H}_{0}\right)$ and $\left(\mathbf{E}_{1}, \mathbf{H}_{1}\right)$. The probes are modelled as currents with sinusoidal shape with unknown amplitude and phase. This probe model is of course an approximation; however, as has been demonstrated in [21], MAS can be used to accurately recover impedance properties of wire antennas, e.g., such probes. Even with this approximate probe model, the present MAS model yields useful results, as will be apparent.

The probes produce an incident field $\left(\mathbf{E}_{1}^{\text {inc }}, \mathbf{H}_{1}^{\text {inc }}\right)$ in Region 1 inside the DRA, whose interaction with the DRA boundary $B$ forms a scattering problem. In Region 1, the total field is thus the sum of the incident and the scattered field $\left(\mathbf{E}_{1}^{\text {scat }}, \mathbf{H}_{1}^{\text {scat }}\right)$. The tangential components of the total field are continuous across $B$, and thus

$$
\begin{aligned}
\hat{\mathbf{n}} \times \mathbf{E}_{0} & =\hat{\mathbf{n}} \times\left(\mathbf{E}_{1}^{\text {inc }}+\mathbf{E}_{1}^{\text {scat }}\right) \\
\hat{\mathbf{n}} \times \mathbf{H}_{0} & =\hat{\mathbf{n}} \times\left(\mathbf{H}_{1}^{\text {inc }}+\mathbf{H}_{1}^{\text {scat }}\right) .
\end{aligned}
$$

In the MAS probe model, the sinusoidal probe current is taken as

$$
\mathbf{I}^{(p)}(z)=\hat{\mathbf{z}} I_{0}^{(p)} \frac{\sin k_{1}(l-z)}{\sin k_{1} l}, \quad 0 \leq z \leq l
$$

where $l$ is the probe length and $I_{0}^{(p)}$ is the complex excitation. The excitations will be calculated based on the scattering matrix of the DRA elements or arrays as detailed in Section II-C.

In order to calculate the incident field, the probe currents are discretized using $Q$ so-called incidence sources (ISs) for each probe. The ISs are electric Hertzian dipoles, and the position and dipole moment of the $q$ th IS of the $p$ th probe are denoted by $\mathbf{r}_{p q}=\left(\rho_{p}, \phi_{p}, z_{q}\right)$ and $\mathbf{p}_{q}^{(p)}=(l / Q) \mathbf{I}^{(p)}\left(z_{q}\right)$, where $z_{q}=$ 
$q l / Q$, respectively. The ISs are shown in Fig. 2(a) and radiate in a homogeneous half-space with the material parameters of Region 1; however, the thus produced incident field is confined to Region 1 . The contribution to the incident field radiated by the $q$ th IS is denoted $\mathbf{E}_{q}^{\mathrm{inc},(p)}$. The incident field radiated by the $p$ th probe is then

$$
\mathbf{E}^{\mathrm{inc},(p)}=\sum_{q=1}^{Q} \mathbf{E}_{q}^{\mathrm{inc},(p)} .
$$

To recover the fields in both regions, two sets of auxiliary sources (ASs) are positioned on so-called auxiliary surfaces denoted $A$ and $D$ and chosen conformal to $B$. The auxiliary surfaces are receded into and advanced outside $B$ by the distances $d_{A}$ and $d_{D}$, respectively, as indicated in Fig. 2(a). The ASs on $A$ and $D$ radiate in homogenous half-spaces with the material parameters of Regions 0 and 1, respectively, and the radiated fields are confined to the respective regions. The ASs are chosen as pairs of crossed Hertzian dipoles of either electric (EHD) or magnetic (MHD) type with independent excitations. The numbers of ASs on $A$ and $D$ are equal and denoted by $N$. The positions of the ASs are denoted by $\mathbf{a}_{n}$ and $\mathbf{d}_{n}$, respectively. On the scatterer surface $B$ the boundary conditions (1a-b) are tested in $M=N$ test points (TPs) at positions $\mathbf{b}_{m}$, and thus the positions of the ASs and TPs are related through

$$
\begin{aligned}
& \mathbf{a}_{n}=\mathbf{b}_{m}-d_{A} \hat{\mathbf{n}}\left(\mathbf{b}_{m}\right), \quad m=n \\
& \mathbf{d}_{n}=\mathbf{b}_{m}+d_{D} \hat{\mathbf{n}}\left(\mathbf{b}_{m}\right), \quad m=n .
\end{aligned}
$$

The total field on the two sides of the boundary $B$, evaluated at the $m$ th TP $\mathbf{b}_{m}$, is

$$
\begin{aligned}
\left\{\begin{array}{l}
\mathbf{E}\left(\mathbf{b}_{m}\right) \\
\mathbf{H}\left(\mathbf{b}_{m}\right)
\end{array}\right\}= & \sum_{n=1}^{N} \sum_{t=1}^{2} C_{n t}^{A}\left\{\begin{array}{l}
\mathbf{E}_{n t}^{A}\left(\mathbf{b}_{m}\right) \\
\mathbf{H}_{n t}^{A}\left(\mathbf{b}_{m}\right)
\end{array}\right\}, \text { Region 0 } \\
\left\{\begin{array}{l}
\mathbf{E}\left(\mathbf{b}_{m}\right) \\
\mathbf{H}\left(\mathbf{b}_{m}\right)
\end{array}\right\}= & \sum_{p=1}^{P}\left\{\begin{array}{l}
\mathbf{E}^{\text {inc },(p)}\left(\mathbf{b}_{m}\right) \\
\mathbf{H}^{\text {inc },(p)}\left(\mathbf{b}_{m}\right)
\end{array}\right\} \\
& +\sum_{n=1}^{N} \sum_{t=1}^{2} C_{n t}^{D}\left\{\begin{array}{l}
\mathbf{E}_{n t}^{D}\left(\mathbf{b}_{m}\right) \\
\mathbf{H}_{n t}^{D}\left(\mathbf{b}_{m}\right)
\end{array}\right\}, \text { Region 1. }
\end{aligned}
$$

In (5a) $\mathbf{E}_{n t}^{A}$ and $\mathbf{H}_{n t}^{A}$ denote the electric and magnetic fields radiated by the $t$ th of the two crossed Hertzian dipoles of the $n$th AS on the auxiliary surface $A$ and similarly for $\mathbf{E}_{n t}^{D}$ and $\mathbf{H}_{n t}^{D}$ in (5b). These fields are weighted by the MAS excitation coefficients $C_{n t}^{A}$ and $C_{n t}^{D}$, which are to be determined through fulfilment of (1a) and (1b) in the $M$ TPs. This yields $4 M$ equations with $4 N$ unknowns for the tangential components of the electric and magnetic fields at $B$. The presence of the infinite PEC ground plane is taken into account by employing image theory. It thus follows that the fields from the ASs and ISs can be found by removing the ground plane and adding the field from the corresponding image source below the ground plane. Thus the field from a single AS above the infinite ground plane consists of two contributions: one from the AS directly and one from the image source, e.g.,

$$
\mathbf{E}_{n t}^{A}\left(\mathbf{b}_{m}\right)=\mathbf{E}_{n t}^{A, \text { direct }}\left(\mathbf{b}_{m}\right)+\mathbf{E}_{n t}^{A, \text { image }}\left(\mathbf{b}_{m}\right) .
$$

Explicit expressions for the electric and magnetic fields, evaluated at the TPs, are given in Appendix II.

In the case where there is more than one probe, i.e., $P>1$, it is convenient to form $P$ independent equation systems with individual sets of incident fields and corresponding solutions of the MAS excitation coefficients. The solutions of these systems may then be combined later in accordance with the actual excitations of the probes. In this way, it is only necessary to invert the linear system matrix once. The linear system of equations thus established can be written as a matrix equation of the form

$$
\left[\begin{array}{l}
\overline{\overline{\mathbf{Z}}}_{E}^{A} \overline{\overline{\mathbf{Z}}}_{E}^{D} \\
\overline{\overline{\mathbf{Z}}}_{H}^{A} \overline{\overline{\mathbf{Z}}}_{H}^{D}
\end{array}\right]\left[\begin{array}{l}
\overline{\overline{\mathbf{C}}}^{A} \\
\overline{\overline{\mathbf{C}}}^{D}
\end{array}\right]=\left[\begin{array}{l}
\overline{\overline{\mathbf{V}}}_{E} \\
\overline{\overline{\mathbf{V}}}_{H}
\end{array}\right] .
$$

The submatrices $\overline{\overline{\mathbf{Z}}}_{E}^{A}, \overline{\overline{\mathbf{Z}}}_{E}^{D}, \overline{\overline{\mathbf{Z}}}_{H}^{A}$, and $\overline{\overline{\mathbf{Z}}}_{H}^{D}$ have $2 M \times 2 N$ elements and hold two tangential components of the electric or magnetic fields of the two Hertzian dipoles of the $N$ ASs on the auxiliary surfaces $A$ or $D$ at the $M$ TPs. The right-hand side submatrices $\overline{\overline{\mathbf{V}}}_{E}$ and $\overline{\mathbf{V}}_{H}$ have $2 M \times P$ elements, and their columns hold the incident electric and magnetic fields from the $P$ probes. The MAS excitation coefficients in $\overline{\mathbf{C}}^{A}$ and $\overline{\overline{\mathbf{C}}}$ are readily found by inversion of the left-hand side matrix and are similarly arranged in columns corresponding to the $P$ sets of incident fields.

\section{B. The DRA Array}

The single-element formulation is now extended to the case of a planar array consisting of $R$ DRAs. Thus the DRA boundaries, regions, auxiliary surfaces, ASs, and TPs are now referred to by an index from one to $R$.

In Fig. 2(b), an example of an MAS configuration with $R=2$ is shown. It is noted that the interaction between the ASs, ISs, and TPs within each DRA element of the array is the same as in the single-element case. The interaction between the ASs and ISs of one DRA element and the TPs of a neighboring DRA elements must, however, now be included. Thus the field radiated by the ASs on the external auxiliary surfaces, denoted by $D r$, as well as the incident field radiated by the ISs inside the $r$ th DRA are confined to Region $r$. Hence it is only the ASs on the internal auxiliary surfaces, denoted by $A r$, that contributes to the field in Region 0 and hence to the field at the boundaries of the other DRA elements. In total a $4 R N$-dimensional linear system of equations results. In the case where the $p$ th probe is excited, the total fields on the two sides of the boundary $\mathrm{Br}$ at the $m$ th TP of the $r$ th DRA $\mathbf{b}_{m}^{r}$ is

$$
\left\{\begin{array}{l}
\mathbf{E}\left(\mathbf{b}_{m}^{r}\right) \\
\mathbf{H}\left(\mathbf{b}_{m}^{r}\right)
\end{array}\right\}=\sum_{s=1}^{R} \sum_{n=1}^{N} \sum_{t=1}^{2} C_{n t}^{A s,(p)}\left\{\begin{array}{l}
\mathbf{E}_{n t}^{A s}\left(\mathbf{b}_{m}^{r}\right) \\
\mathbf{H}_{n t}^{A s}\left(\mathbf{b}_{m}^{r}\right)
\end{array}\right\}
$$

in Region 0 and

$$
\begin{aligned}
\left\{\begin{array}{c}
\mathbf{E}\left(\mathbf{b}_{m}^{r}\right) \\
\mathbf{H}\left(\mathbf{b}_{m}^{r}\right)
\end{array}\right\}= & \sum_{p=1}^{R P} \gamma_{p r}\left\{\begin{array}{l}
\mathbf{E}^{\mathrm{inc},(p)}\left(\mathbf{b}_{m}^{r}\right) \\
\mathbf{H}^{\mathrm{inc},(p)}\left(\mathbf{b}_{m}^{r}\right)
\end{array}\right\} \\
& +\sum_{n=1}^{N} \sum_{t=1}^{2} C_{n t}^{D r,(p)}\left\{\begin{array}{c}
\mathbf{E}_{n t}^{D r}\left(\mathbf{b}_{m}^{r}\right) \\
\mathbf{H}_{n t}^{D r}\left(\mathbf{b}_{m}^{r}\right)
\end{array}\right\}
\end{aligned}
$$

in Region $r$. The factor $\gamma_{p r}$ takes the value 1 if the $p$ th probe is located in the $r$ th region and 0 otherwise. Thus for each element 
the summation over the $R P$ probes only yields $P$ contributions. Note that the sum in (8a) includes the interior ASs of all the $R$ DRAs, whereas those of (8b) only include the ISs and exterior ASs associated with the $r$ th DRA. The corresponding matrix systems now becomes

$$
\begin{aligned}
& {\left[\begin{array}{cccc}
\overline{\overline{\mathbf{Z}_{1}^{1}}} & \overline{\overline{\mathbf{Z}_{1}^{2}}} & \ldots & \overline{\overline{\mathbf{Z}_{1}^{\mathbf{R}}}} \\
\overline{\overline{\mathbf{Z}_{2}^{1}}} & \overline{\overline{\mathbf{Z}_{2}^{2}}} & \ldots & \overline{\overline{\mathbf{Z}_{2}^{\mathbf{R}}}} \\
\vdots & \vdots & \ddots & \vdots \\
\overline{\overline{\mathbf{Z}_{\mathbf{R}}^{1}}} & \overline{\overline{\mathbf{Z}_{\mathbf{R}}^{2}}} & \cdots & \overline{\overline{\mathbf{Z}_{\mathbf{R}}^{\mathbf{R}}}}
\end{array}\right]\left[\begin{array}{cccc}
\overline{\overline{\mathbf{C}_{1}^{1}}} & \overline{\overline{\mathbf{C}_{1}^{2}}} & \cdots & \overline{\overline{\mathbf{C}_{1}^{\mathbf{R}}}} \\
\overline{\overline{\mathbf{C}_{2}^{1}}} & \overline{\overline{\mathbf{C}_{2}^{2}}} & \ldots & \overline{\overline{\mathbf{C}_{2}^{\mathbf{R}}}} \\
\vdots & \vdots & \ddots & \vdots \\
\overline{\overline{\mathbf{C}_{\mathbf{R}}^{1}}} & \overline{\overline{\mathbf{C}_{\mathbf{R}}^{2}}} & \cdots & \overline{\overline{\mathbf{C}_{\mathbf{R}}^{\mathbf{R}}}}
\end{array}\right]} \\
& =\left[\begin{array}{cccc}
\overline{\overline{\mathbf{V}_{1}^{\mathbf{1}}}} & \overline{\overline{\mathbf{0}}} & \cdots & \overline{\overline{\mathbf{0}}} \\
\overline{\overline{\mathbf{0}}} & \overline{\overline{\mathbf{V}_{2}^{2}}} & \cdots & \overline{\overline{\mathbf{0}}} \\
\vdots & \vdots & \ddots & \vdots \\
\overline{\overline{\mathbf{0}}} & \overline{\overline{\mathbf{0}}} & \cdots & \overline{\overline{\mathbf{V}_{\mathbf{R}}^{\mathrm{R}}}}
\end{array}\right] \text {. }
\end{aligned}
$$

The submatrices $\overline{\overline{\mathbf{V}_{\mathbf{j}}^{\mathbf{i}}}}, \overline{\overline{\mathbf{C}_{\mathbf{j}}^{\mathbf{i}}}}$, and $\overline{\overline{\mathbf{Z}_{\mathbf{j}}^{\mathbf{i}}}}$ in (9) are of the same size as the full matrices for the single-element case in (7). In particular, it is noted that $\overline{\overline{\mathbf{Z}_{\mathbf{j}}^{\mathbf{i}}}}$ equals the left-hand side matrix in (7) for $i=j$. The $P$ columns of $\overline{\overline{\mathbf{V}_{\mathbf{j}}^{\mathbf{i}}}}$ and $\overline{\overline{\mathbf{C}_{\mathbf{j}}^{\mathbf{i}}}}$ hold the incident fields, and MAS coefficients associated with the $j$ th DRA corresponding to the case where the $P$ probes of the $i$ th DRA are excited. Since the incident field is confined inside the respective DRA, the $\overline{\overline{\mathbf{V}_{\mathbf{j}}^{\mathbf{i}}}}$ are only nonzero for $i=j$. However, since the neighboring DRA becomes excited due to the mutual coupling between the DRAs, the $\overline{\overline{\mathbf{C}_{\mathbf{j}}^{\mathbf{j}}}}$ are generally nonzero. The full $\overline{\overline{\mathbf{C}}}$ and $\overline{\overline{\mathbf{V}}}$ matrices have $R P$ columns corresponding to each of the $R P$ probes in the array.

\section{Calculation of Impedances and Far Fields}

The probe input ports are located at the probes' intersections with the ground plane. The self and mutual impedances of these ports are calculated using the reaction theorem [23]. In the present model, where the probe currents are discretized using $Q$ ISs, a discrete version of the reaction theorem is employed

$$
Z_{i j}=-\frac{1}{I_{0}^{(i)} I_{0}^{(j)}} \sum_{q=1}^{Q} \mathbf{E}^{(i)}\left(\mathbf{r}_{j q}+\hat{\boldsymbol{\rho}} \rho_{0}\right) \cdot \mathbf{p}_{q}^{(j)}
$$

where $\mathbf{E}^{(i)}\left(\mathbf{r}_{j q}+\hat{\boldsymbol{\rho}} \rho_{0}\right)$ is the field sampled at the surface of the $j$ th probe when the $i$ th probe is excited. The electric field is calculated as

$$
\begin{aligned}
\mathbf{E}^{(i)}\left(\mathbf{r}_{j q}+\hat{\boldsymbol{\rho}} \rho_{0}\right)= & \sum_{r=1}^{R} \gamma_{j r}\left[\gamma_{i r} \mathbf{E}^{\mathrm{inc},(i)}\left(\mathbf{r}_{j q}+\hat{\boldsymbol{\rho}} \rho_{0}\right)\right. \\
& \left.+\sum_{n=1}^{N} \sum_{t=1}^{2} C_{n t}^{D r,(i)} \mathbf{E}_{n t}^{D r}\left(\mathbf{r}_{j q}+\hat{\boldsymbol{\rho}} \rho_{0}\right)\right] .
\end{aligned}
$$

The factor $\gamma_{j r} \gamma_{i r}$, multiplied on the incident field, indicates that this only contributes directly to the coupling between probes located in the same region.
In this model the probes are assumed to be excited with forward propagating voltage waves $\mathbf{V}^{+}$. The input reflection coefficient $\Gamma_{\text {in }, p}$ and input impedance $Z_{\text {in }, p}=R_{\text {in }, p}+j X_{\text {in }, p}$ of the $p$ th port are calculated from

$$
\begin{aligned}
\Gamma_{\text {in }, p} & =\frac{V_{p}^{-}}{V_{p}^{+}} \\
Z_{\text {in }, p} & =Z_{0} \frac{1+\Gamma_{\text {in }, p}}{1-\Gamma_{\text {in }, p}}
\end{aligned}
$$

where $Z_{0}=50 \Omega$ is the assumed characteristic impedance of the feed lines connected to the probe ports. The reflected voltage waves $V_{p}^{-}$are given by

$$
\begin{aligned}
\mathbf{V}^{-} & =\overline{\overline{\mathbf{S}}} \mathbf{V}^{+} \\
\overline{\overline{\mathbf{S}}} & =\left(\overline{\overline{\mathbf{Z}}}+Z_{0} \overline{\overline{\mathbf{U}}}\right)^{-1}\left(\overline{\overline{\mathbf{Z}}}-Z_{0} \overline{\overline{\mathbf{U}}}\right) .
\end{aligned}
$$

In (13b), $\overline{\overline{\mathbf{Z}}}$ is the impedance matrix with the elements given by (10) and $\overline{\overline{\mathrm{U}}}$ is the identity matrix. While the amplitudes of the probes currents were set to unity when calculating the probe impedances, the actual probe excitations, corresponding to a certain set of forward voltage wave excitations $\mathbf{V}^{+}$, can now be calculated using the obtained knowledge of the coupling between the probes.

The field outside the DRAs, i.e., in Region 0 , is the sum of the contributions from the ASs on the interior auxiliary surfaces $A r$. Thus the field in Region 0 due to a current on the $p$ th probe is

$$
\mathbf{E}_{0}^{(p)}(\mathbf{r})=\sum_{r=1}^{R} \sum_{n=1}^{N} \sum_{t=1}^{2} C_{n t}^{A r,(p)} \mathbf{E}_{n t}^{A r}(\mathbf{r})
$$

From this expression and the knowledge of the element-to-element coupling, the active element patterns (AEPs) [13] of the array can be found as follows. When any of the probe ports are excited by a forward voltage wave, nonzero currents will result on all the probes of the array due to the coupling between the probes and hence all DRA elements are excited. In the case where the $p^{*}$ th port is excited by a unit forward voltage wave, the corresponding current of the $p$ th probe is

$$
I_{0}^{\left(p, p^{*}\right)}=\frac{V_{p}^{+}-V_{p}^{-}}{Z_{0}}, V_{p}^{+}= \begin{cases}1 \mathrm{~V}, & \text { for } p=p^{*} \\ 0, & \text { otherwise }\end{cases}
$$

where $V_{p}^{-}$follow from (13a). Having calculated the currents on all probes, the corresponding AEPs can be established. For a unit forward voltage wave excitation of $1 \mathrm{~V}$ of the $p^{*}$ th port, the resulting AEPs is

$$
\mathbf{E}_{\mathrm{AEP}}^{\left(p^{*}\right)}(\mathbf{r})=\sum_{p=1}^{R P} W_{p, p^{*}} \mathbf{E}_{0}^{(p), f a r}(\mathbf{r})
$$

where $\mathbf{E}_{0}^{(p), f a r}$ is the far field corresponding to $\mathbf{E}_{0}^{(p)}$ of (14) and $W_{p, p^{*}}=I_{0}^{\left(p, p^{*}\right)} / I_{0}^{(p)}$ are dimensionless weight factors. 
TABLE I

THE SiX INVESTIGATED DESIGNS OF THE HEMISPHEROIDAL DRA ELEMENT

\begin{tabular}{|c|c|c|c|c|c|c|c|c|}
\hline Design & $\begin{array}{c}h \\
{[\mathrm{~mm}]}\end{array}$ & $\begin{array}{c}w \\
{[\mathrm{~mm}]}\end{array}$ & $\epsilon_{r}$ & $\begin{array}{c}\rho_{p} \\
{[\mathrm{~mm}]}\end{array}$ & $\begin{array}{c}l \\
{[\mathrm{~mm}]}\end{array}$ & $\begin{array}{c}f_{\text {res }} \\
{[\mathrm{GHz}]}\end{array}$ & $\begin{array}{c}\text { BW } \\
{[\%]}\end{array}$ & $N$ \\
\hline 0 & 20 & 40 & 22 & 13 & 10.0 & 1.54 & 2.7 & 165 \\
\hline 1 & 20 & 50 & 18 & 15 & 11.0 & 1.49 & 4.0 & 223 \\
\hline 2 & 20 & 60 & 15 & 18 & 12.1 & 1.49 & 6.5 & 223 \\
\hline 3 & 20 & 70 & 13 & 21 & 13.0 & 1.49 & 8.1 & 223 \\
\hline 4 & 20 & 80 & 12 & 23 & 13.5 & 1.48 & 12.9 & 297 \\
\hline 5 & 20 & 90 & 11 & 24 & 14.1 & 1.49 & 14.7 & 297 \\
\hline
\end{tabular}

The array patterns for specific beam scanning follow straightforwardly by applying suitable weights $W_{p^{*}}^{+}\left(\theta_{0}, \phi_{0}\right)$ for the forward voltage waves exciting the ports, corresponding to the desired scan angles $\left(\theta_{0}, \phi_{0}\right)$. Thus the array far field is

$$
\mathbf{E}_{\text {array }}^{\text {far }}\left(\theta_{0}, \phi_{0}, \mathbf{r}\right)=\sum_{p^{*}=1}^{R P} W_{p^{*}}^{+}\left(\theta_{0}, \phi_{0}\right) \mathbf{E}_{\mathrm{AEP}}^{\left(p^{*}\right)}(\mathbf{r}) .
$$

\section{ANALYSIS OF DRA ELEMENTS}

The MAS model is now applied to the case of a single DRA element. The investigations presented here will concentrate on oblate hemispheroidal DRA elements, i.e., $w \geq 2 h$. The elements are circularly polarized with $P=4$ probes. The probe displacement $\rho_{p}$ from the center is chosen such that the probes are positioned as close as possible to the DRA edge and excite the fundamental broadside mode [1]. The probe displacement $\rho_{p}$ is the same for all probes but varies for different DRA designs. The probes are spaced equiangularly such that $\phi_{p}=90^{\circ}(p-1)$ and ideal phase quadrature is imposed for the forward voltage waves of the ports, i.e., $V_{p}^{+}=(-j)^{p-1} \mathrm{~V}$. The frequency interval of interest is L-band around 1.6 GHz. Six different designs, denoted Design 0-5, are considered and are further described in Table I, where also the number of ASs on each auxiliary surface $N$ is given. For all the designs, the probe height $l$ has been selected such that $k_{1} l=\pi / 2$ at $1.6 \mathrm{GHz}$, and a probe radius of $\rho_{0}=0.5 \mathrm{~mm}$ and element height of $h=2 \mathrm{~cm}$ are used throughout. Furthermore $\mu_{r}=1$, and by varying $w$ and $\epsilon_{r}$, the resonance frequency $f_{\text {res }}$, defined at $X_{\mathrm{in}}=0$, can be tuned. Investigations, which are not shown here, indicate that the input impedance of the DRA elements, and in particular the center element, is shifted to slightly higher frequencies when used in an array. For this reason, the single-element designs presented have values of $\epsilon_{r}$ selected to obtain a resonance frequency somewhat lower than $1.6 \mathrm{GHz}$, as can also be seen from Table I.

In order to check the convergence of the solution, the relative changes $\Delta_{Z}^{\mathrm{IS}}\left(Q_{n}\right), \Delta_{Z}^{\mathrm{AS}}\left(N_{n}\right)$ of the input impedance, resulting from an increase in the number of ISs and ASs, are calculated. $\Delta_{Z}^{\mathrm{IS}}\left(Q_{n}\right)$ is defined as

$$
\Delta_{Z}^{\mathrm{IS}}\left(Q_{n}\right)=\frac{\left|Z_{\text {in }}\left(Q_{n}\right)-Z_{\text {in }}\left(Q_{n-1}\right)\right|}{\left|Z_{\text {in }}\left(Q_{n-1}\right)\right|}
$$

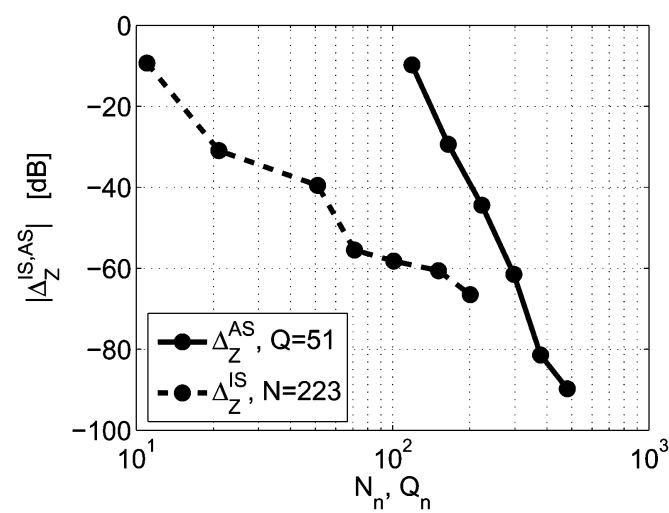

Fig. 3. Convergence of the input impedance for increasing $Q$ and $N$ for Design 1.

where $N=223$ is kept constant. For $\Delta_{Z}^{\mathrm{AS}}\left(N_{n}\right)$, which is defined in a similar way, $Q=51$ is kept constant. The results are shown in Fig. 3 for Design 1. As can be seen, the change quickly becomes very small indicating convergence. In the remaining investigations, $Q=71$ is used throughout. As a further validation, the MAS model is compared with results obtained using SWE and CST-MS.

Design 0 , with $w=2 h$, is the special case of a hemispherical DRA. The probe-fed hemispherical DRA has been widely described in the literature, and selected analytical solutions are reported in [1] and [2]. An analytical solution, based on a spherical wave expansion, has been derived here (see Appendix I), and this serves as an additional means for testing the proposed MAS model. In Fig. 4, the input impedance and directivity obtained for Design 0 are shown together with the corresponding SWE and CST-MS results. It is noted that the MAS probe model is employed for both the MAS and the SWE solutions but not for the CST-MS solution. In the CST-MS model, the probes are modelled more accurately with coaxial cable feed ports, which might not lead to the sinusoidal current distribution assumed in the MAS model. As can be seen the MAS and SWE results agree very well for both impedance and radiation results. This shows that the field both inside and outside the DRA is accurately recovered. When comparing the impedance results with the CST-MS solution, it is seen that the approximate probe model used in the SWE and MAS models introduces some inaccuracy. Thus the resonance frequency is about $2 \%$ higher and the resistance at resonance is about $20 \%$ lower than for CST-MS. In the case of Design 0, MAS yields a resistance of about $80 \Omega$ while the CST-MS result is about $100 \Omega$. For the radiation results, however, all three solutions agree well and the probe models have little impact on the result.

To calculate the impedance bandwidth, the DRA input ports are matched with identical lossless open-circuit single-stub (OCSS) matching networks as shown in Fig. 5. This matching network is designed to match the mean input reflection coefficient of the $P$ probe ports

$$
\Gamma_{\mathrm{in}}^{E}\left(f_{0}\right)=\frac{1}{P} \sum_{p=1}^{P} \Gamma_{\mathrm{in}, p}\left(f_{0}\right)
$$

where $\Gamma_{\mathrm{in}, p}\left(f_{0}\right)$ is the input reflection coefficient, seen at the $p$ th probe input port of the unmatched DRA element, and is calculated from (12a) for a chosen design frequency $f_{0}$. The 

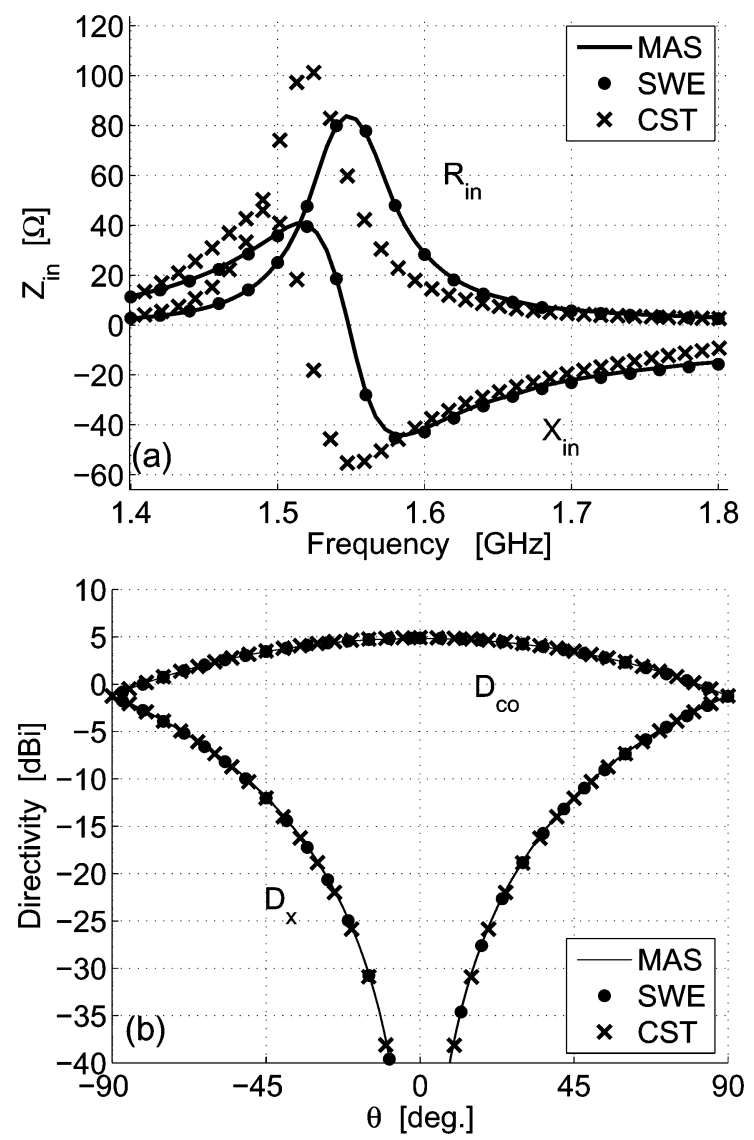

Fig. 4. Comparison of the results for the hemispherical Design 0 calculated by MAS and the SWE solution. (a) Input impedance $Z_{\text {in. }}$. (b) Directivity for $f=1.6 \mathrm{GHz}$.

scattering matrix of the OCSS matching network can easily be determined and is

$$
\begin{aligned}
\overline{\overline{\mathbf{S}}}_{\mathrm{OCSS}}(f)= & \frac{1}{2+j \tan \left(\beta d_{1}\right)} \\
& \times\left[\begin{array}{cc}
-j \tan \left(\beta d_{1}\right) & 2 e^{-j \beta d_{2}} \\
2 e^{-j \beta d_{2}} & -j \tan \left(\beta d_{1}\right) e^{-2 j \beta d_{2}}
\end{array}\right]
\end{aligned}
$$

where $d_{1}, d_{2}$, and $\beta$ are the lengths and phase constant of the transmission lines in the matching network. The proper values of $d_{1}$ and $d_{2}$ depend on the reflection coefficient to be matched. The design frequency $f_{0}$ and expressions can be found in most textbooks on the topic, e.g., [24]. By combining the scattering parameters of the matching network $\overline{\overline{\mathbf{S}}}_{\text {OCSS }}$ with those of the array in (13b), e.g., as described in [25], the combined scattering matrix of the matched DRA element can be derived and the reflected voltages from this matched DRA can be calculated. The matching network is designed for $f_{0}=1.5 \mathrm{GHz}$, which is close to the element resonance frequencies $f_{\text {res }}$, and the impedance bandwidth (BW) is defined with respect to $\left|\hat{\Gamma}_{\mathrm{in}}^{E}(f)\right|^{2}<-10 \mathrm{~dB}$, where

$$
\left|\hat{\Gamma}_{\mathrm{in}}^{E}(f)\right|^{2}=\frac{1}{P} \sum_{p=1}^{P}\left|\hat{\Gamma}_{\mathrm{in}, p}(f)\right|^{2}
$$

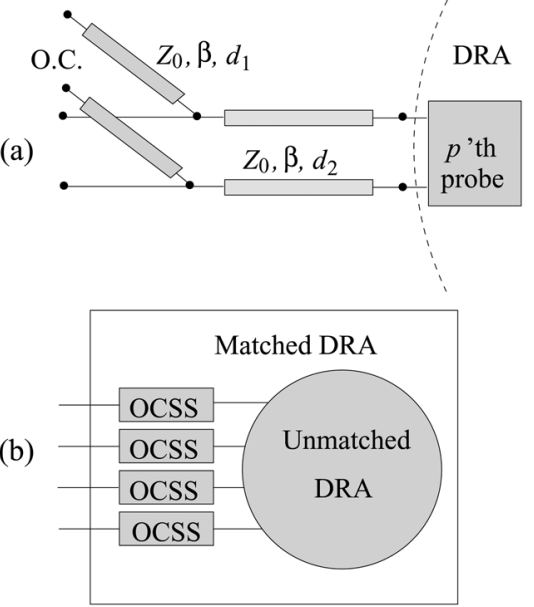

Fig. 5. (a) OCSS matching network connected to a probe port. (b) A matched DRA with four probes and matching elements.

and $\hat{\Gamma}_{\mathrm{in}, p}(f)$ is the input reflection coefficient seen at the input port of the OCSS matching network connected to the $p$ th probe.

The impedance bandwidths and resonance frequencies obtained with the MAS model are listed in Table I for all the designs. The impedance results for Design 1, 3 , and 5 are shown in Fig. 6 and compared with the corresponding CST-MS results. The deviations between the resonance frequencies and impedance values obtained by the two solutions are still approximately $2 \%$ higher and $20 \%$ lower, respectively, for the MAS solutions. The impact of increasing $w$ and decreasing $\epsilon_{r}$ on the bandwidth is clearly seen, and thus the obtained impedance bandwidths range from $2.7 \%$ to $14.7 \%$ around $1.5 \mathrm{GHz}$. It is also seen that the impedances become smaller as the permittivity is decreased. For Design 1 and 5, examples of radiation patterns for three frequencies are shown in Fig. 7. It is seen that the radiation patterns are quite similar. This illustrates the fact that the shape of the DRA does not influence the radiation very much since it is the same fundamental broadside mode that is excited. It should be noted that the high level of cross-polarization near the horizon is a consequence of the infinite ground plane used in the model and would not appear to the same extent for a finite ground plane.

\section{ANALYSIS OF DRA ARRAYS}

In this section, the MAS model is applied to a seven-element phased array where the identical elements are positioned in a hexagonal lattice and are separated by the distance $d$, as shown in Fig. 8. The hexagonal lattice is advantageous compared to the rectangular lattice for phased array applications since wider element separations can be used before the scan angle dependent effects of grating lobes and impedance mismatch become too severe [13]. Also the hexagonal lattice improves the rotational symmetry of the radiation pattern compared to the rectangular one.

However, before applying the MAS model to the entire sevenelement array, the MAS and CST-MS results are compared for a simple two-element configuration. In this way, the MAS model can be validated for the case where more than one element is 

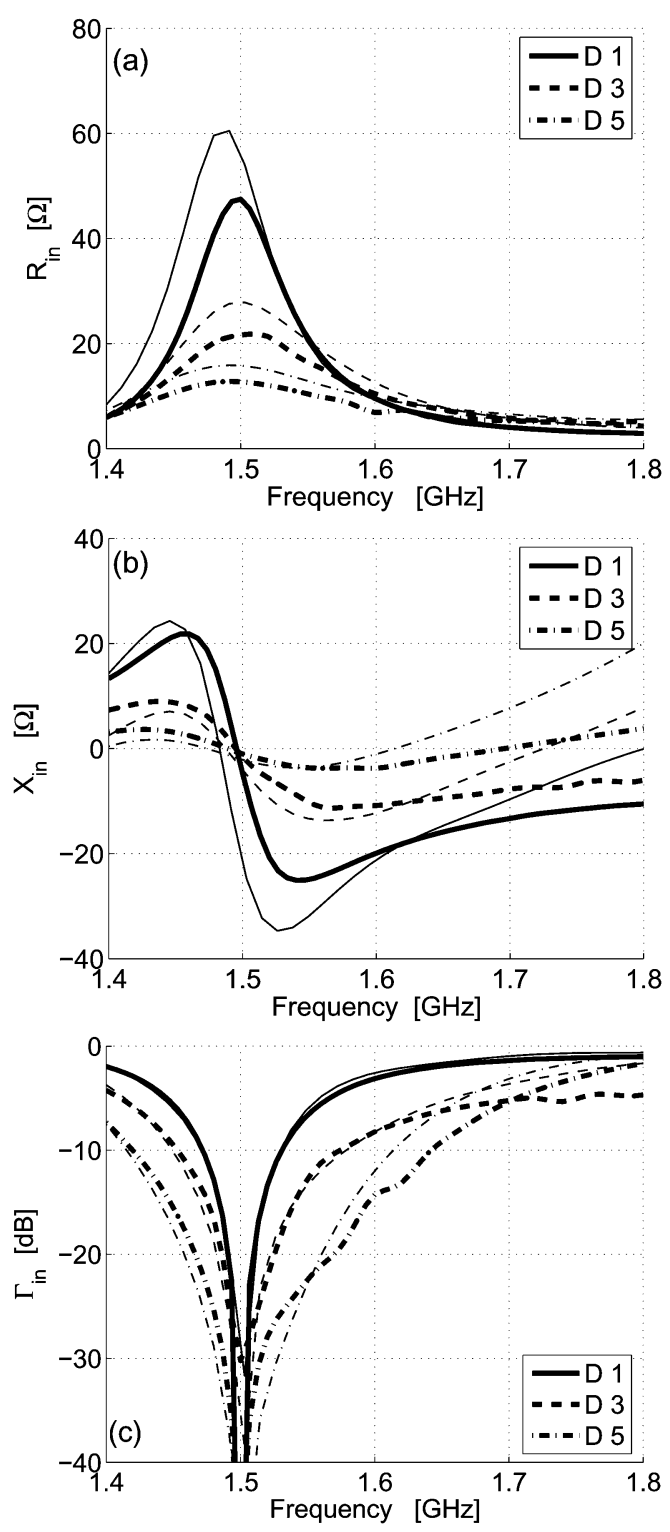

Fig. 6. Impedance results for probe 1 of Designs 1, 3, and 5 for the MAS (thick lines) and CST-MS (thin lines) solutions. (a) Input resistance $R_{\mathrm{in}, 1}$. (b) Input reactance $X_{\text {in }, 1}$. (c) Reflection coefficient $\hat{\Gamma}_{\text {in }}^{E}(f)$ after matching with the OCSS matching network designed with $f_{0}=1.5 \mathrm{GHz}$.

present. With reference to Fig. 8, the investigated two-element configuration corresponds to the case where only elements 1 and 2 are present. The elements are displaced by $d=9 \mathrm{~cm}$. In Fig. 9 (a) and (b), the mutual impedances of port 1 (i.e., $p=1$ ) in element 1 and ports 1,2 , and 3 in element 2 are shown as a function of frequency. In Fig. 9(c), the directivity resulting from an excitation of port 1 of element 2 is shown. Again it is seen that the radiation results are accurately recovered by the MAS model. However, due to the approximate probe model, the mutual impedances are somewhat lower than predicted by CST-MS

To further validate the method, reference results from [22] of the mutual coupling between two single-probe hemispherical DRA elements is reproduced. The DRA elements have the parameters $w=2 h=50.8 \mathrm{~mm}, \epsilon_{r}=9.5, l=6.5 \mathrm{~mm}$,
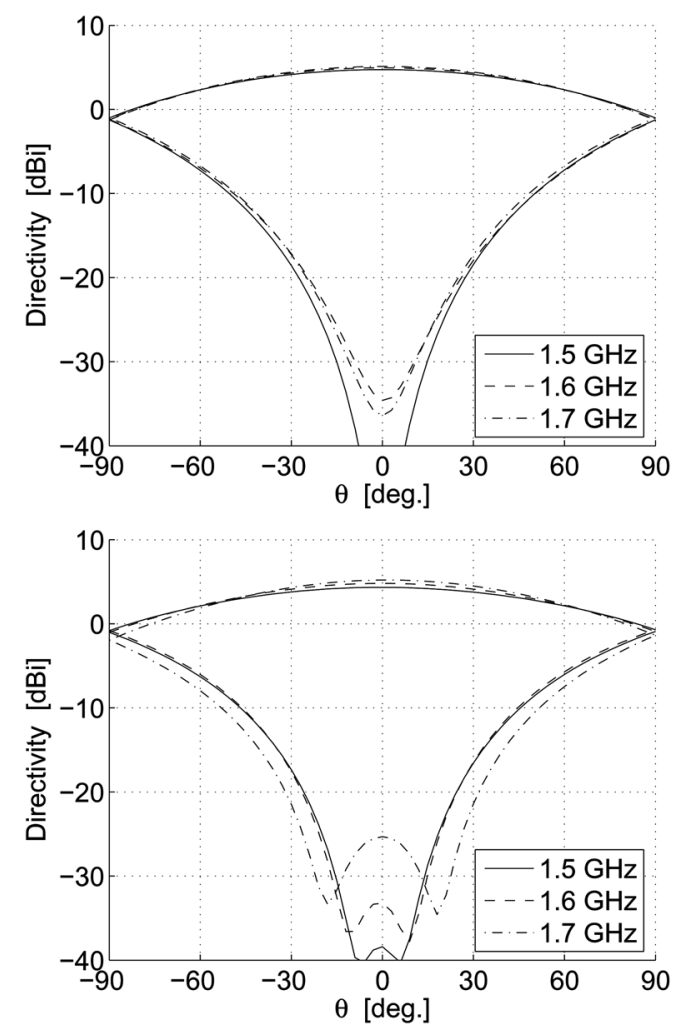

Fig. 7. Directivity for DRA elements calculated with the MAS model. Both DRAs are matched for $f_{0}=1.5 \mathrm{GHz}$. (a) Design 1, MAS. (b) Design 5 .

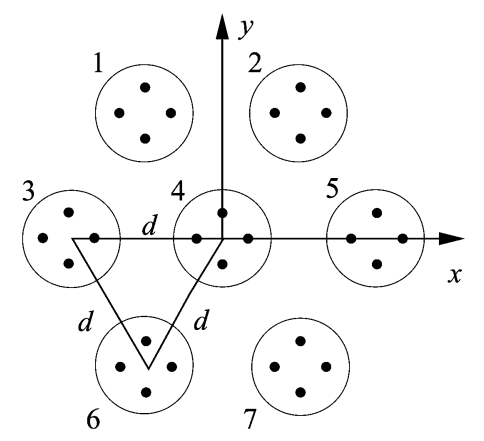

Fig. 8. Top view of the DRA array with element numbering.

$\rho_{p}=6.4 \mathrm{~mm}$, and $\rho_{0}=0.5 \mathrm{~mm}$. The measurements were performed at a frequency of $3.84 \mathrm{GHz}$. In Fig. 10, the MAS results are compared with the measured reference results. It is seen that the agreement is very good with only minor deviations in the E-plane for a separation of about one wavelength.

Having validated the MAS model, the analysis of the phased array is now resumed. The elements of the array are matched with OCSS matching networks, designed for $f_{0}=1.6 \mathrm{GHz}$. The choice of which reflection coefficient to match is, however, less obvious for several reasons. First, the presence of neighboring elements has direct impact on the self- and mutual impedances of the probe ports. Secondly, the coupling between the elements causes scan-dependent variation in the input impedances. Lastly, the frequency dependence of the impedances should be considered.

In order to ensure that the employed matching network does not favor a specific scan direction, it has been chosen not to 

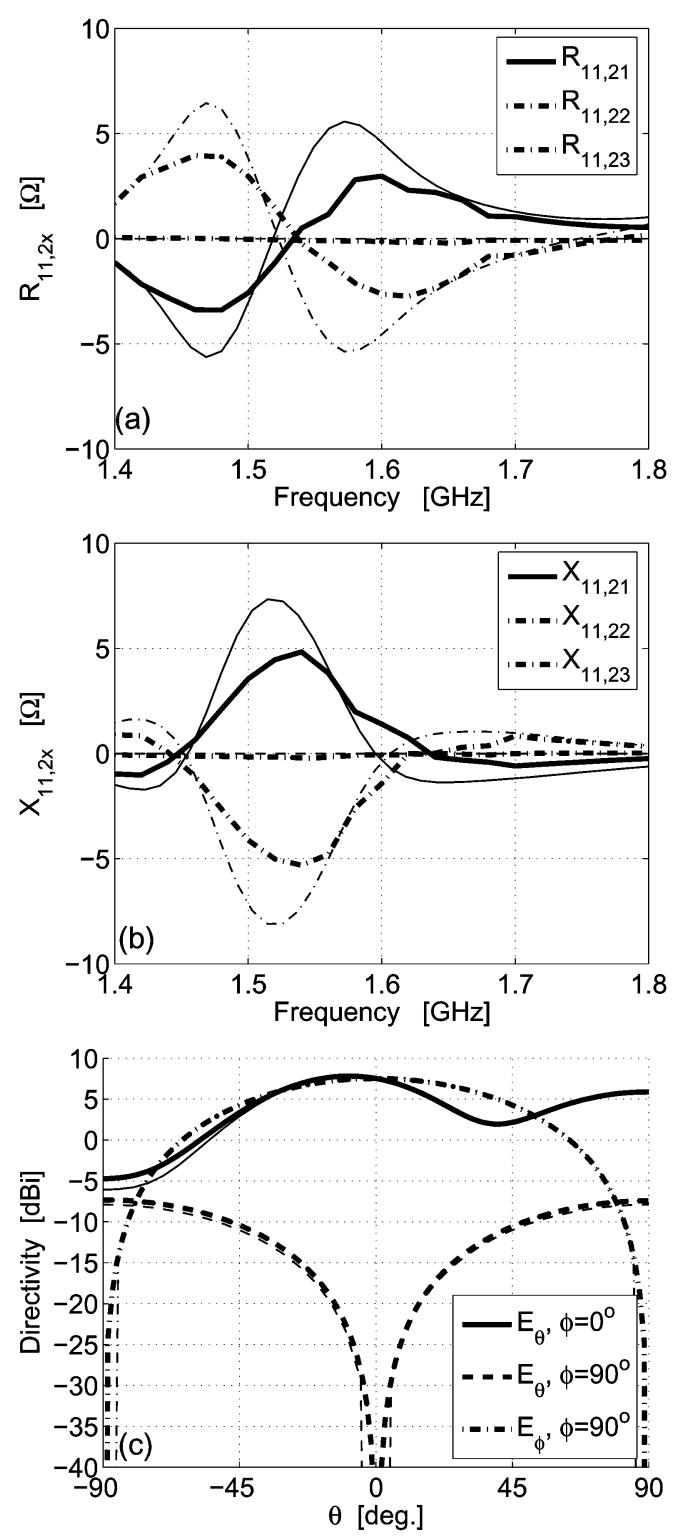

Fig. 9. Results for two-element configuration calculated by MAS (thick lines) and CST-MS (thin lines). The double indexes refer to element number and probe number as indicated in Figs. 1 and 8. (a) Mutual resistance. (b) Mutual reactance. (c) Radiation pattern when port 1 of element 1 is excited.

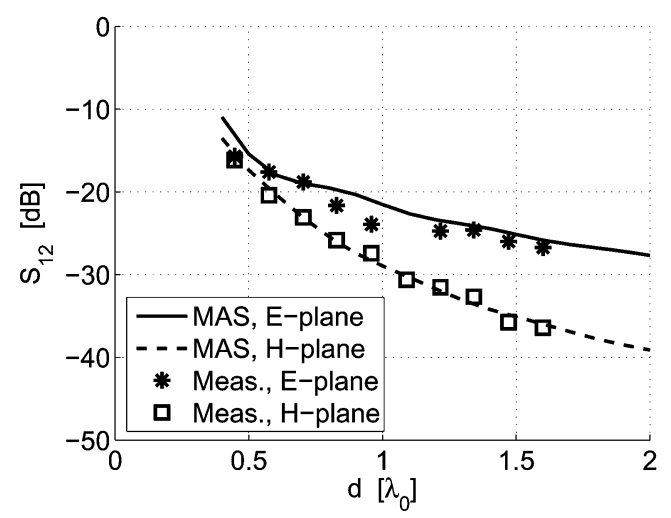

Fig. 10. Mutual coupling in the $E$ - and $H$-plane between two single-probe hemispherical DRA elements as a function of element separation $d$ in free-space wavelengths $\lambda_{0}$. The calculated MAS results are compared with measurements from [22].
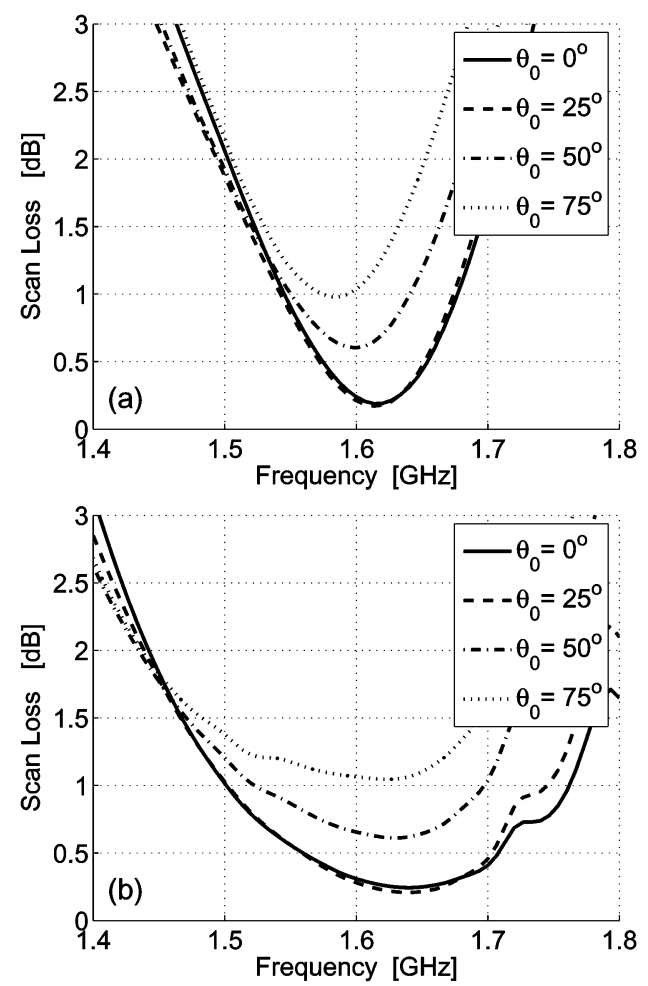

Fig. 11. Resulting scan loss of the hemispheroidal DRA array when matched with the OCSS matching network. (a) Design 1. (b) Design 3.

include the element-to-element coupling when designing the matching network. Therefore $R$ mean reflection coefficients $\Gamma_{\mathrm{in}, r}^{E}$ are calculated, one for each of the unmatched DRA elements, in the same way as in (19). These reflection coefficients vary somewhat from element to element, and therefore an average between the elements is used. Furthermore, the frequency variation is taken into account by averaging the $\Gamma_{\mathrm{in}, r}^{E}$ over frequency. The OCSS matching networks for the array are thus designed to match the reflection coefficient

$$
\Gamma_{\mathrm{in}}^{A}=\frac{1}{f_{2}-f_{1}} \frac{1}{R} \int_{f=f_{1}}^{f_{2}} \sum_{r=1}^{R} \Gamma_{\mathrm{in}, r}^{E}(f) d f
$$

where $f_{1}=1.52 \mathrm{GHz}$ and $f_{2}=1.66 \mathrm{GHz}$. In a manner similar to the single element case, the reflection coefficients $\hat{\Gamma}_{\mathrm{in}, p}\left(\theta_{0}, \phi_{0}, f\right)$ seen at the input port of the OCSS matching networks are calculated.

The definition of the scan loss used in this paper only includes the effects of impedance mismatch. Although the main beam directivity also varies with scan angle, this is not included in the scan loss but treated separately. The scan loss is thus defined as

$$
S L\left(\theta_{0}, \phi_{0}, f\right)=\frac{1}{1-\left|\hat{\Gamma}_{\mathrm{in}}^{A}\left(\theta_{0}, \phi_{0}, f\right)\right|^{2}}
$$

where $\left|\hat{\Gamma}_{\mathrm{in}}^{A}\left(\theta_{0}, \phi_{0}, f\right)\right|^{2}$ is defined similar to (21), with $P$ being replaced with $R P$. Examples of the calculated scan loss are shown in Fig. 11 for a DRA array with Design 1 and 3 elements. The element separation is $d=9 \mathrm{~cm}$ in both cases, which equals $0.48 \lambda_{0}$ at $f=1.6 \mathrm{GHz}$. The shown results are the worst case for all azimuthal scan angles $\phi_{0}$ as a function of $\theta_{0}$ and frequency. 

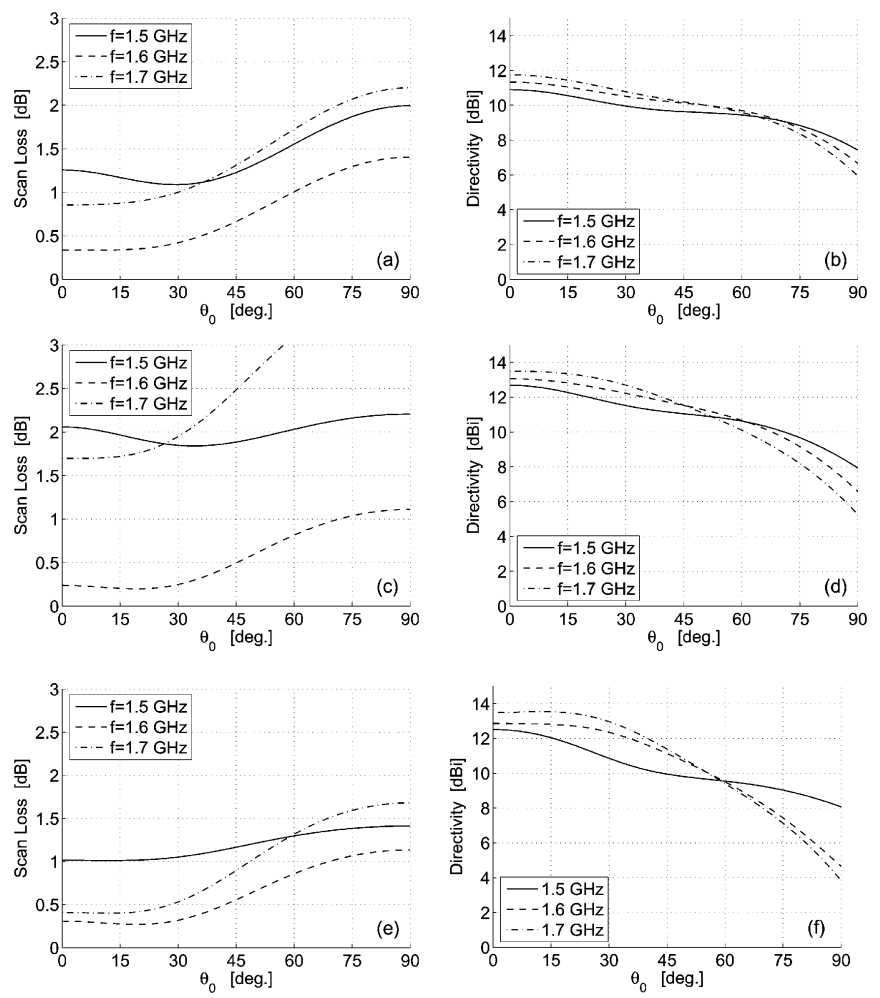

Fig. 12. Resulting scan loss and main beam copolar directivity of the hemispheroidal DRA array for Designs 1 and 3. (a) and (b) Design 1 with $d=7 \mathrm{~cm}$. (c) and (d) Design 1 with $d=9 \mathrm{~cm}$. (e) and (f) Design 3 with $d=9 \mathrm{~cm}$.

The impact of the element bandwidth is evident since the broadband Design 3 has low scan loss for a larger frequency interval than the narrow-band Design 1. Also the impact of scanning the beam towards large $\theta_{0}$ angles is clear and the scan loss generally increases with $\theta_{0}$. For $f=1.6 \mathrm{GHz}$, the increase is from about $0.25 \mathrm{~dB}$ for $\theta_{0} \leq 25^{\circ}$ to about $1 \mathrm{~dB}$ for $\theta_{0} \simeq 75^{\circ}$ for the two cases.

In Fig. 12, the variation of the scan loss and main beam directivity with scan angle is shown for Designs 1 and 3, the former with two different values of the element separation $d=7 \mathrm{~cm}$ $\left(0.37 \lambda_{0}\right.$ at $\left.f=1.6 \mathrm{GHz}\right)$ and $d=9 \mathrm{~cm}$. The general tendency of increasing scan loss for increasing $\theta_{0}$ is seen in all cases, but the impact of the element separation is particularly noteworthy. For Design 1, with closely spaced elements, a relatively low scan loss is obtained for the two extreme frequencies 1.5 and 1.7 GHz, whereas significantly higher scan loss results for the larger separation. With respect to the main beam directivity, it is seen that it is more uniform with respect to scan angle for the small separation than for large. This illustrates the positive impact on both scan loss and directivity due to closely positioned elements. In the case of Design 3, the element width $w=7 \mathrm{~cm}$ precludes such close element separation and $d=9 \mathrm{~cm}$ is used. With respect to the main beam directivity, however, the large variation with $\theta_{0}$ persists and even deteriorates.

\section{CONCLUSION}

The method of auxiliary sources has been applied for the modelling and analysis of circularly polarized probe-fed hemispheroidal dielectric resonator antenna elements and phased ar- rays. The MAS solutions were compared with the simulation tool CST Microwave Studio, measured reference results, and in the case of a hemispherical DRA also with a spherical wave expansion solution. The agreement is excellent as far as the radiation results are concerned; however, the MAS probe model implies a slight deviation of about $2 \%$ in the obtained resonance frequencies and the resistances at resonance are about $20 \%$ smaller for MAS than the CST-MS results. For the SWE solution, where the same probe model is used, the impedance results agree excellently. This demonstrates that MAS can be used to effectively analyze even complicated antennas with high accuracy.

Investigations were carried out for different element designs where the DRA height was kept at $2 \mathrm{~cm}$ and the element width and permittivity were varied to maintain a resonance frequency somewhat below $1.6 \mathrm{GHz}$. The resulting impedance bandwidths ranged between $2.7 \%$ for the hemispherical case to $14.7 \%$ for a 9-cm-wide DRA.

Two designs were subsequently selected for use in a sevenelement hexagonal phased array. The impact of the element impedance bandwidths and separation on the resulting scan loss and main beam directivity were investigated as a function of frequency and scan angles. As expected, the wide DRA elements yielded the lowest scan loss due to their inherently large bandwidth. It was, however, demonstrated that lowering the element separation, which is possible for the small elements, has a positive impact on the array bandwidth and serves to lower the scan loss. Furthermore, the main beam directivity was more uniform with respect to scan angle for the smaller element separation.

This analysis has been carried out assuming an infinite ground plane beneath the DRA. This limitation may, however, be overcome by including a finite ground plane in the MAS model as proposed in [26].

\section{APPENDIX I}

\section{ANALYTICAL RESULTS FOR HEMISPHERICAL DRA}

The hemispherical DRA on an infinite ground plane can be modelled as a dielectric sphere in free space with all current sources being augmented by appropriate image sources below the ground plane. For this dielectric sphere, the dyadic Green's function $\underline{\mathrm{G}}^{E}\left(\mathbf{r}, \mathbf{r}^{\prime}\right)$ for the electric field has been derived and can be expressed as an SWE. The electric field everywhere then follows from

$$
\mathbf{E}(\mathbf{r})=\int_{V} \underline{\underline{\mathbf{G}}}^{E}\left(\mathbf{r}, \mathbf{r}^{\prime}\right) \cdot \mathbf{J}\left(\mathbf{r}^{\prime}\right) d V^{\prime}
$$

where $\mathbf{J}\left(\mathbf{r}^{\prime}\right)$ is the current source inside the DRA including the image source. The dyadic Green's function can be expressed as

$$
\underline{\underline{\mathbf{G}}}^{E}\left(\mathbf{r}, \mathbf{r}^{\prime}\right)=k_{1}^{2} Z_{1} \sum_{s=1}^{2} \sum_{n=1}^{\infty} \sum_{m=-n}^{n} \underline{\underline{g}}_{s m n}^{E}\left(\mathbf{r}, \mathbf{r}^{\prime}\right)
$$

where

$$
\begin{aligned}
\underline{\underline{\mathbf{g}}}_{s m n}^{E}= & (-1)^{m}\left[\mathbf{F}_{s,-m, n}^{(4)}\left(k_{1}, \mathbf{r}^{\prime}\right)+A_{s n} \mathbf{F}_{s,-m, n}^{(1)}\left(k_{1}, \mathbf{r}^{\prime}\right)\right] \\
& \times \mathbf{F}_{s m n}^{(1)}\left(k_{0}, \mathbf{r}\right), \quad r<r^{\prime}
\end{aligned}
$$




$$
\begin{aligned}
\mathbf{E}_{n t}^{A, \text { direct }}\left(\mathbf{v}_{n m}\right)=E_{0} \frac{e^{-j k_{0} r_{n m}^{v}}}{k_{0} r_{n m}^{v}}\left[\frac{2}{k_{0} r_{n m}^{v}}\left[1-\frac{j}{k_{0} r_{n m}^{v}}\right] \hat{\mathbf{v}}_{n m}^{r}\left[f_{1} \sin \theta_{n m}^{v}+f_{3} \cos \theta_{n m}^{v}\right]\right. \\
\left.-\left[j+\frac{1}{k_{0} r_{n m}^{v}}-\frac{j}{\left(k_{0} r_{n m}^{v}\right)^{2}}\right]\left[\hat{\mathbf{v}}_{n m}^{\theta}\left(\cos \theta_{n m}^{v} f_{1}-f_{3} \sin \theta_{n m}^{v}\right)-f_{2} \hat{\mathbf{v}}_{n m}^{\phi}\right]\right] \\
\mathbf{H}_{n t}^{A, \operatorname{direct}}\left(\mathbf{v}_{n m}\right)=j \frac{E_{0}}{Z_{0}} \frac{e^{-j k_{0} r_{n m}^{v}}}{k_{0} r_{n m}^{v}}\left[1-\frac{j}{k_{0} r_{n m}^{v}}\right]\left[\hat{\mathbf{v}}_{n m}^{\phi}\left[f_{3} \sin \theta_{n m}^{v}-f_{1} \cos \theta_{n m}^{v}\right]-\hat{\mathbf{v}}_{n m}^{\theta} f_{2}\right]
\end{aligned}
$$

$$
\begin{aligned}
& \underline{\underline{\mathrm{g}}}_{s m n}^{E}=(-1)^{m} \mathbf{F}_{s,-m, n}^{(1)}\left(k_{1}, \mathbf{r}^{\prime}\right) \\
& \times\left[A_{s n} \mathbf{F}_{s m n}^{(1)}\left(k_{1}, \mathbf{r}\right)+\mathbf{F}_{s m n}^{(4)}\left(k_{1}, \mathbf{r}\right)\right], \quad a>r>r^{\prime} \\
& \underline{\underline{\mathrm{g}}}_{s m n}^{E}=(-1)^{m} B_{s n} \mathbf{F}_{s,-m, n}^{(1)}\left(k_{1}, \mathbf{r}^{\prime}\right) \mathbf{F}_{s m n}^{(4)}\left(k_{0}, \mathbf{r}\right), \quad r>a \text {. }
\end{aligned}
$$

$\mathbf{F}_{\text {smn }}^{(1,4)}$ are spherical vector wave functions, $a$ is the radius of the dielectric sphere, and

$$
\begin{aligned}
A_{s n}= & -\frac{1}{\Delta_{s n}}\left[R_{s n}^{(4)}\left(k_{1} a\right) R_{3-s, n}^{(4)}\left(k_{0} a\right)\right. \\
& \left.\quad-\frac{k_{1} \mu_{0}}{k_{0} \mu_{1}} R_{3-s, n}^{(4)}\left(k_{1} a\right) R_{s n}^{(4)}\left(k_{0} a\right)\right] \\
B_{s n}= & \frac{j(-1)^{s+1}}{\Delta_{s n}} \\
\Delta_{s n}= & R_{s n}^{(1)}\left(k_{1} a\right) R_{3-s, n}^{(4)}\left(k_{0} a\right) \\
& -\frac{k_{1} \mu_{0}}{k_{0} \mu_{1}} R_{3-s, n}^{(1)}\left(k_{1} a\right) R_{s n}^{(4)}\left(k_{0} a\right) .
\end{aligned}
$$

The functions $\mathbf{F}_{s m n}^{(1,4)}$ and $R_{s n}^{(1,4)}$ used in (26a)-(26c) and (27a)-(27c) are all defined in [27]. In the case where EHD is used to model the probe currents, the integral in (24) simplifies to a summation.

\section{APPENDIX II}

\section{AUXILIARY SOURCE FIELDS AT TEST POINTS}

Presently, only the ASs on $A$ are considered. The same principle applies for the ASs on $D$. The unit vectors of the local coordinate systems of the $n$th AS on $A$ and the $m$ th TP are denoted by $\left(\hat{\mathbf{a}}_{n}^{x}, \hat{\mathbf{a}}_{n}^{y}, \hat{\mathbf{a}}_{n}^{z}\right)$ and $\left(\hat{\mathbf{b}}_{n}^{x}, \hat{\mathbf{b}}_{n}^{y}, \hat{\mathbf{b}}_{n}^{z}\right)$, respectively. Thus the two orthogonal Hertzian dipoles of the $n$th AS coincide with $\hat{\mathbf{a}}_{n}^{x}$ for $t=1$ in (5) and $\hat{\mathbf{a}}_{n}^{y}$ for $t=2 . \hat{\mathbf{b}}_{n}^{z}$ coincides with $\hat{\mathbf{n}}\left(\mathbf{b}_{m}\right)$. In order to express the field from the $n$th AS in terms of the coordinate system of the $m$ th TP, a rotated version of the $n$th AS coordinate system is introduced. It is rotated through the Euler angles $\left(\chi_{0}, \theta_{0}, \phi_{0}\right)$ [27] such that its rectangular unit vectors, denoted by $\left(\hat{\mathbf{v}}_{n m}^{x}, \hat{\mathbf{v}}_{n m}^{y}, \hat{\mathbf{v}}_{n m}^{z}\right)$, are parallel to $\left(\hat{\mathbf{b}}_{n}^{x}, \hat{\mathbf{b}}_{n}^{y}, \hat{\mathbf{b}}_{n}^{z}\right)$. The corresponding spherical unit vectors are denoted by $\left(\hat{\mathbf{v}}_{n m}^{r}, \hat{\mathbf{v}}_{n m}^{\theta}, \hat{\mathbf{v}}_{n m}^{\phi}\right)$ and the position of the $m$ th TP, described in this coordinate system, is denoted by $\mathbf{v}_{n m}$ with the rectangular and spherical coordinates $\left(x_{n m}^{v}, y_{n m}^{v}, z_{n m}^{v} ; r_{n m}^{v}, \theta_{n m}^{v}, \phi_{n m}^{v}\right)$. Expressed in this coordinate system, the electric field from, e.g., the $t$ th Hertzian dipole of the $n$th AS on the interior auxiliary surface $A$, evaluated at the $m$ th TP $\mathbf{b}_{m}$, on $B$ is

$$
\left[\begin{array}{c}
\hat{\mathbf{b}}_{m}^{x} \cdot \mathbf{E}_{n t}^{A}\left(\mathbf{b}_{m}\right) \\
\hat{\mathbf{b}}_{m}^{y} \cdot \mathbf{E}_{n t}^{A}\left(\mathbf{b}_{m}\right) \\
\hat{\mathbf{b}}_{m}^{z} \cdot \mathbf{E}_{n t}^{A}\left(\mathbf{b}_{m}\right)
\end{array}\right]=\overline{\overline{\mathbf{T}}}\left(\theta_{n m}^{v}, \phi_{n m}^{v}\right)\left[\begin{array}{c}
\hat{\mathbf{v}}_{n m}^{r} \cdot \mathbf{E}_{n t}^{A}\left(\mathbf{v}_{n m}\right) \\
\hat{\mathbf{v}}_{n m}^{\theta} \cdot \mathbf{E}_{n t}^{A}\left(\mathbf{v}_{n m}\right) \\
\hat{\mathbf{v}}_{n m}^{\phi} \cdot \mathbf{E}_{n t}^{A}\left(\mathbf{v}_{n m}\right)
\end{array}\right]
$$

and similarly for the magnetic field. Here

$$
\overline{\overline{\mathbf{T}}}(\theta, \phi)=\left[\begin{array}{ccc}
\sin \theta \cos \phi & \cos \theta \cos \phi & -\sin \phi \\
\sin \theta \sin \phi & \cos \theta \sin \phi & \cos \phi \\
\cos \theta & -\sin \theta & 0
\end{array}\right]
$$

converts from spherical to rectangular coordinates. In the case where the ASs are chosen as EHD, the direct electric and magnetic fields are given by (30a) and (30b), shown at the top of the page, where $E_{0}=1(\mathrm{~V} / \mathrm{m})$. For $t=1$

$$
\begin{aligned}
f_{1}= & \cos \theta_{0} \cos \phi_{0} \cos \left(\chi_{0}+\phi_{n m}^{v}\right) \\
& -\sin \phi_{0} \sin \left(\chi_{0}+\phi_{n m}^{v}\right) \\
f_{2}= & \sin \phi_{0} \cos \left(\chi_{0}+\phi_{n m}^{v}\right) \\
& +\cos \theta_{0} \cos \phi_{0} \sin \left(\chi_{0}+\phi_{n m}^{v}\right) \\
f_{3}= & \sin \theta_{0} \cos \phi_{0}
\end{aligned}
$$

and for $t=2, \phi_{0}$ should be replaced with $\phi_{0}-(\pi / 2)$ in (30c)-(30e). The coordinates of $\mathbf{v}_{n m}$ are given by

$$
\begin{aligned}
x_{n m}^{v} & =\hat{\mathbf{b}}_{m}^{x} \cdot\left(\mathbf{b}_{m}-\mathbf{a}_{n}\right) \\
y_{n m}^{v} & =\hat{\mathbf{b}}_{m}^{y} \cdot\left(\mathbf{b}_{m}-\mathbf{a}_{n}\right) \\
z_{n m}^{v} & =\hat{\mathbf{b}}_{m}^{z} \cdot\left(\mathbf{b}_{m}-\mathbf{a}_{n}\right) .
\end{aligned}
$$

In the case where MHDs are used as ASs, the corresponding fields can be found by application of the duality principle. The direct field from (30a)-(30b) should be augmented by the field from the image source beneath the infinite ground plane. This image source field can be calculated in the same way as the direct field by using the image position

$$
\mathbf{a}_{n}^{\mathbf{i}}=\mathbf{a}_{n}-2 \hat{\mathbf{z}}\left(\hat{\mathbf{z}} \cdot \mathbf{a}_{n}\right)
$$

where $\hat{\mathbf{z}}$ is perpendicular to the infinite ground plane, and local unit vectors

$$
\left(\hat{\mathbf{a}}_{n}^{\mathbf{i} x}, \hat{\mathbf{a}}_{n}^{\mathbf{i} y}, \hat{\mathbf{a}}_{n}^{\mathbf{i} z}\right)=\alpha\left(-\hat{\mathbf{a}}_{n}^{x},-\hat{\mathbf{a}}_{n}^{y}, \hat{\mathbf{a}}_{n}^{z}\right)
$$


where $\alpha=1$ and $\alpha=-1$ should be chosen for the EHD and MHD ASs, respectively, in order to be in accordance with the "+" of (6). For the ASs on the exterior auxiliary surface $D$, the $k_{0}$ and $Z_{0}$ in (30a) and (30b) should be replaced by $k_{1}$ and $Z_{1}$.

\section{REFERENCES}

[1] K. W. Leung, K. M. Luk, K. Y. A. Lai, and D. Lin, "Theory and experiment of a coaxial probe fed hemispherical dielectric resonator antenna," IEEE Trans. Antennas Propag., vol. 41, no. 10, pp. 1390-1398, 1993.

[2] Z. N. Chen, K. W. Leung, K. M. Luk, and E. K. N. Yung, "Electromagnetic scattering from the probe-fed dielectric resonator antenna," in Proc. IEEE Int. Symp. Antennas Propag., 1996, vol. 2, pp. 1410-1413.

[3] S. A. Long, M. W. McAllister, and L. C. Shen, "The resonant cylindrical dielectric cavity antenna," IEEE Trans. Antennas Propag., vol. AP-31, no. 3, pp. 406-412, 1983.

[4] M. Cooper, A. Petosa, A. Ittipiboon, and J. S. Wight, "Investigation of dielectric resonator antennas for L-band communications," in Proc. Symp. Antenna Technol. Appl. Electromagn., 1996, pp. 167-170.

[5] A. Ittipiboon, A. Petosa, D. Roscoe, and M. Cuhaci, "An investigation of a novel broadband dielectric resonator antenna," in Proc. IEEE Int. Symp. Antennas Propag., 1996, vol. 3, pp. 2038-2041.

[6] K.-L. Wong, N.-C. Chen, and H.-T. Chen, "Analysis of an hemispherical dielectric resonator antenna with an air gap," IEEE Microw. Guided Wave Lett., vol. 3, pp. 355-357, 1993.

[7] A. A. Kishk, R. Chair, and K. F. Lee, "Broadband dielectric resonator antennas excited by L-shaped probe," IEEE Trans. Antennas Propag., vol. 54, no. 8, pp. 2182-2189, 2006.

[8] A. A. Kishk, X. Zhang, A. W. Glisson, and D. Kajfez, "Numerical analysis of stacked dielectric resonator antennas excited by a coaxial probe for wideband applications," IEEE Trans. Antennas Propag., vol. 51, no. 8, pp. 1996-2006, 2003.

[9] A. A. Kishk, A. W. Glisson, and G. P. Junker, "Bandwidth enhancement for split cylindrial dielectric resonator antennas," Progr. Electromagn. Res., vol. 33, pp. 97-118, 2001.

[10] K. W. Leung and H. K. Ng, "Theory and experiment of circularly polarized dielectric resonator antenna with a parasitic patch," IEEE Trans. Antennas Propag., vol. 51, no. 3, pp. 405-411, 2003.

[11] M. B. Oliver, Y. M. M. Antar, R. K. Mongia, and A. Ittipiboon, "Circularly polarised rectangular dielectric resonator antenna," Electron. Lett., vol. 31, no. 6, pp. 418-419, 1995.

[12] G. Drossos, Z. Wu, and L. E. Davis, "Circular polarised cylindrical dielectric resonator antenna," Electron. Lett., vol. 32, no. 4, pp. 281-283, 1996.

[13] R. J. Mailloux, Phased Array Antenna Handbook. Boston, MA: Artech House, 1994

[14] A. A. Kishk, "Dielectric resonator antenna elements for array applications," in Proc. IEEE Int. Symp. Phased Array Syst. Technol., 2003, pp. 300-305.

[15] A. Petosa, R. Larose, A. Ittipiboon, and M. Cuhaci, "Low profile phased array of dielectric resonator antennas," in Proc. IEEE Int. Symp. Phased Array Syst. Technol., 1996, pp. 182-185.

[16] A. Petosa, A. Ittipiboon, and M. Cuhaci, "Array of circularly polarised cross dielectric resonator antennas," Electron. Lett., vol. 32, no. 19, pp. 1742-1743, 1996.

[17] S. P. Skobelev, "Algorithm of the method of auxiliary sources for analysis of arrays of circular waveguides with protruding dielectric rods," in Proc. IEEE Int. Symp. Phased Array Syst. Technol., 2003, pp. 333-338.

[18] D. I. Kaklamani and H. T. Anastassiu, "Aspects of the method of auxiliary sources in computational electromagnetics," IEEE Antennas Propag. Mag., vol. 44, no. 3, pp. 48-64, 2002.

[19] S. Eisler and Y. Leviatan, "Analysis of electromagnetic scattering from metallic and penetrable cylinders with edges using a multifilament current model," Proc. Inst. Elect. Eng., vol. 6, pt. H, pp. 431-438, 1989.

[20] N. V. Larsen and O. Breinbjerg, "A hybrid MAS/MoM technique for 2 D impedance scatterers illuminated by closely positioned sources," Microw. Opt. Technol. Lett., vol. 44, no. 2, pp. 112-114, 2005.

[21] P. J. Papakanellos and C. N. Capsalis, "Numerical analysis of cylindrical dipole antennas using an auxiliary sources model," J. Electromagn. Waves Appl., 2003.
[22] K.-M. Luk, W.-K. Leung, and K.-W. Leung, "Mutual impedance of hemispherical dielectric resonator antennas," IEEE Trans. Antennas Propag., vol. 42, no. 12, pp. 1652-1654, 1994.

[23] R. F. Harrington, Time-Harmonic Electromagnetic Fields, Classical Reissue. New York: Wiley, 2001.

[24] D. M. Pozar, Microwave Engineering, 2nd ed. New York: Wiley, 1998.

[25] J. A. Dobrowolski, Introduction To Computer Methods For Microwave Circuit Analysis And Design, 2nd ed. New York: Wiley, 1998.

[26] N. V. Larsen and O. Breinbjerg, "Modelling the impact of ground planes on antenna radiation using the method of auxiliary sources," IET Microw. Antennas Propag. 2007.

[27] J. E. Hansen, Ed., Spherical Near-Field Antenna Measurements. London, U.K.: Peregrinus, 1988.

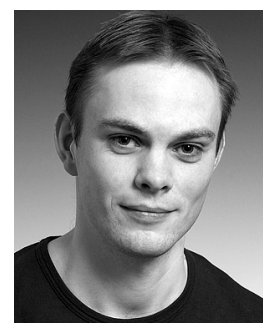

Niels Vesterdal Larsen was born in Copenhagen, Denmark, on February 7, 1977. He received the M.Sc. degree in electrical engineering from the Technical University of Denmark, Lyngby, in 2004, where he is working toward the Ph.D. degree.

In the summer and fall of 2006, he was a Visiting Scholar with the Jet Propulsion Laboratory, Pasadena, CA. He is currently with Thrane \& Thrane, Kgs. Lyngby, Denmark. His research interests include antennas, phased arrays, electromagnetic field theory, and computational electromagnetics.

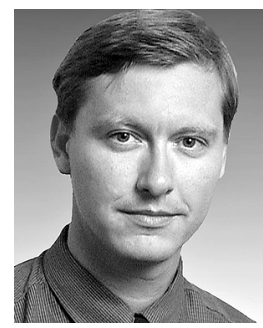

Olav Breinbjerg ( $\left.M^{\prime} 87\right)$ was born in Silkeborg, Denmark, on July 16, 1961. He received the M.Sc. and $\mathrm{Ph} . \mathrm{D}$. degrees in electrical engineering from the Technical University of Denmark (DTU), Lyngby, in 1987 and 1992, respectively.

He has since been on the Faculty of Ørsted, DTU, (formerly the Department of Electromagnetic Systems and Electromagnetics Institute), where he is now a full Professor. Since 1997, he has been Head of the Antenna and Electromagnetics Group and, since 2000, the Head of the DTU-ESA Spherical Near-Field Antenna Test Facility. Since 2001, he has been Coordinator of the DTU Electrical Engineering Line, and since 2006 he is the Programme Coordinator for DTU's M.Sc. program in electrical engineering. He was a Visiting Scientist with Rome Laboratory, Hanscom Air Force Base, MA, in fall 1988 and a Fulbright Research Scholar with the University of Texas at Austin, in spring 1995. He has served on several Ph.D. and position assessment committees at universities in Denmark, Sweden, and Finland. He was Danish Representative to the Management Committees of the EU COST-260 Action on Smart Antennas from 1997 to 2001 and the EU COST-284 Action on Innovative Antennas from 2002 to 2006. He is currently Danish Representative to the Management Committee of the EU COST-ICO63 on Antenna Systems and Sensors. He is a Member of the Executive Board of the EU Network of Excellence, Antenna Centre of Excellence, since 2004, a Member of the Danish Strategic Research Council's Programme Committee for Non-Ionizing Radiation since 2004, and a Member of the URSI Danish National Committee since 2004. His research interest is generally in applied electromagnetics-and particularly in antennas, antenna measurements, computational techniques, and scattering-for applications in wireless communication and sensing technologies. Several antenna research and development projects have been undertaken. These led to the DTU-ESA $12 \mathrm{GHz}$ Validation Standard Antenna, the Ørsted Satellite Communication Antenna, and the Rømer Satellite Communication Antenna. At present, his antenna interests focus on application of double-negative materials and phased arrays. He is the author or coauthor of more than 140 journal papers, conference papers, or technical reports. He has taught several B.Sc. and M.Sc. courses in the area of applied electromagnetic field theory on topics such as fundamental electromagnetics, analytical and computational electromagnetics, antennas, and antenna measurements at DTU, where he has also supervised more than 65 special courses, 29 M.Sc. final projects, and seven Ph.D. projects.

Prof. Breinbjerg received a U.S. Fulbright Research Award in 1995 and was awarded the 2001 AEG Elektron Foundation's Award in recognition of his research in applied electromagnetics. He received the 2003 DTU Student Union's Teacher of the Year Award. He is a member of the IEEE Antennas and Propagation Society. 